\title{
Integration by parts and representation of information functionals
}

\author{
Ivan Nourdin \\ Luxembourg University \\ Luxembourg City, Luxembourg \\ Email: ivan.nourdin@uni.lu
}

\author{
Giovanni Peccati \\ Luxembourg University \\ Luxembourg City, Luxembourg \\ Email: giovanni.peccati@uni.lu
}

\author{
Yvik Swan \\ Université de Liège \\ Liège, Belgium \\ Email: yswan@ulg.ac.be
}

\begin{abstract}
We introduce a new formalism for computing expectations of functionals of arbitrary random vectors, by using generalised integration by parts formulae. In doing so we extend recent representation formulae for the score function introduced in [19] and also provide a new proof of a central identity first discovered in [7]. We derive a representation for the standardised Fisher information of sums of i.i.d. random vectors which we use to provide rates of convergence in information theoretic central limit theorems (both in Fisher information distance and in relative entropy) and a Stein bound for Fisher information distance.

Index Terms-Score function, Stein matrix, Fisher information, Representation formulae, Total variation distance.
\end{abstract}

\section{INTRODUCTION}

Let $X$ be a random vector in $\mathbb{R}^{d}$, with differentiable density $f$. The score function $\rho_{X}(x)=$ $\nabla \log f(x)$ has long been known to provide useful handles on the law of $X$. A much less studied object is the Stein matrix of $X$, defined in (2) which can be interpreted as a counterpart to the score where, rather than taking log-derivatives, one considers a special form of integration. This matrix (whose properties when $d=1$ are closely related to the so-called $w$-function, see [5]) has only recently started to attract the attention of the community, see e.g. [18], [19], [12]. We refer the reader to [1] for a detailed study.

In this paper we explore the connexion between the score and the Stein matrix of an arbitrary random vector $X$. Rather than defining these two quantities explicitly in terms of the density $f$, we choose to characterise them by their behaviour through specialised integration by parts formulae (see equations (11) and (2)). Exploiting these we obtain new representation formulae for the Fisher information $J(X)=E\left[\rho_{X}(X) \rho_{X}(X)^{T}\right]$ of an arbitrary random vector $X$ (see Theorem III.3). Such results are akin to those from [7] (see Theorem IV.2 for details) and, more generally, to the classical representation formulae for Fisher information in terms of conditional expectations (see [13] or [16] for a discussion). As an application we obtain (under the assumption that the Stein matrix exists) new information theoretic bounds for Gaussian approximation problems. Our bounds are of the same order as those obtained in the pathbreaking references [8], [2] (in the univariate setting under the assumption of a finite Poincaré constant/spectral gap; see also [3] for multivariate extensions).

Our approach is inspired by results usually exploited within the context of the so-called Stein's method (see [17], [6]). The connexion between Stein's method and Fisher information was discovered in [4] (in the context of compound Poisson approximation) and first studied explicitly by [14], [15] as well as [20]. We conclude the paper in Section $\nabla$ with a new proof of these bounds; our take on these matters does not rely on Stein's method and is of independent interest to the ISIT crowd.

The outline of the paper is as follows. All formulae and definitions are given in Section [II In Section [III we prove the representation formulae for the score in terms of the Stein matrix. In Section IV] we prove a version of the celebrated MMSE formula from [7]. In Section $\nabla$ we provide a general "Stein bound" on the standardised Fisher information of sums of iid random vectors.

\section{SCORE AND STEIN MATRIX}

Fix an integer $d \geq 1$. Let $X, Y$ be centered random $d$-vectors (all elements in $\mathbb{R}^{d}$ are taken as $d \times 1$ column vectors) which we throughout assume to admit a density (with respect to the Lebesgue measure) with support $S \subset \mathbb{R}^{d}$.

Definition II.1. The score of $X$ is the random vector $\rho_{X}(X)$ which satisfies

$$
E\left[\rho_{X}(X) \varphi(X)\right]=-E[\nabla \varphi(X)]
$$


(with $\nabla$ the usual gradient in $\mathbb{R}^{d}$ ) for all test functions $\varphi \in C_{c}^{\infty}\left(\mathbb{R}^{d}\right)$. Any random $d \times d$ matrix $\tau_{X}(X)$ which satisfies

$$
E\left[\tau_{X}(X) \nabla \varphi(X)\right]=E[X \varphi(X)]
$$

for all test functions $\varphi \in C_{c}^{\infty}\left(\mathbb{R}^{d}\right)$ is called a Stein matrix for $X$.

If $X$ has covariance matrix $C$, then a direct application of the definition of the Stein matrix yields $E\left[\tau_{X}(X)\right]=C ; E\left[\rho_{X}(X)\right]=0$ and $E\left[\rho_{X}(X) X^{T}\right]=-I d$, where ${ }^{T}$ denotes the transpose operator and $I d$ is the $d \times d$ identity matrix. For a Gaussian random vector $Z$ with covariance matrix $C$ one uses the well-known Stein identity (see, e.g., [8])

$$
E[Z \varphi(Z)]=C E[\nabla \varphi(Z)]
$$

to prove that $\rho_{Z}(Z)=-C^{-1} Z$ is the score of $Z$ and $\tau_{Z}(Z)=C$ is a Stein matrix of $Z$. Identity (3) characterizes the Gaussian distribution in the sense that a random vector $X$ with support $\mathbb{R}^{d}$ satisfies (3) for all $\varphi \in C_{c}^{\infty}\left(\mathbb{R}^{d}\right)$ if and only if $X$ is itself Gaussian with covariance $C$. More generally, the following result holds (see, e.g., [8]).

Proposition II.2. Let $X$ have density $f$. If $X$ has a score then it is uniquely defined as $\rho_{X}(X)$ with $\rho_{X}(x)=\nabla \log f(x)$.

In the case $d=1$, under standard assumptions of regularity of the density $f$, the existence of the Stein matrix $\tau$ follows from standard integration by parts arguments, from which one deduces that $\tau$ is uniquely defined as $\tau(x)=f(x)^{-1} \int_{x}^{\infty} f(y) d y$. In higher dimensions, the existence of a Stein matrix for $X$ also follows easily from an integration by parts argument, once one can find a matrix valued function $x \mapsto A(x)$ whose components $a_{i j}$ with $1 \leq i, j \leq d$ satisfy $\sum_{j=1}^{d} \frac{\partial}{\partial x_{j}}\left(a_{i j}(x) f(x)\right)=$ $-x_{i}$ for all $i=1, \ldots, d$. As demonstrated in the huge body of literature revolving around Malliavin calculus (see [19] as well as the monograph [17]), a Stein matrix always exists for random vectors that are given by a smooth transformation of a given Gaussian field. Contrarily to the score, however, there is no reason for which the Stein matrix, at least according to our definition, should be unique.

Definition II.3. Let $X$ be a d-random vector with density $f$ and covariance $B$ (invertible), and let $\phi$ be the density of a centered Gaussian random vector $Z$ with covariance $C$. The relative entropy of $X$ is $D(X \| Z)=$ $E[\log (f(X) / \phi(X))]$. The Fisher information (matrix) of $X$ is $J(X)=E\left[\rho_{X}(X) \rho_{X}(X)^{T}\right]$ and its relative Fisher information matrix is $\mathcal{J}(X)=$ $E\left[\left(\rho_{X}(X)+B^{-1} X\right)\left(\rho_{X}(X)+B^{-1} X\right)^{T}\right]$. The standardised Fisher information distance of $X$ is $J_{s t}(X)=\operatorname{tr}(B \mathcal{J}(X))$, with 'tr' the usual trace operator.

Entropy and Fisher information are related to one another via the so-called de Bruijn's identity, see [10. Lemma 2.2] for the original statement, as well as [19, Lemma 2.3] for the forthcoming version.

Lemma II.4 (Multivariate de Bruijn's identity). Let $X$ be a random d-vector with covariance $C$ (invertible) and let $Z$ be Gaussian with covariance $C$ as well. Then $D(X \| Z)=\int_{0}^{1} \frac{1}{2 t} J_{s t}\left(X_{t}\right) d t$.

Remark II.5. There is some confusion surrounding the denomination "de Bruijn's identity" as several different (and not perfectly equivalent) formulations of this identity are available in the literature. See e.g. [7. Section II.D] for an alternative formulation.

\section{REPRESENTATION FORMULAE}

The following lemma is a generalization of [19. Lemma 2.9] to the case of summands with arbitrary distribution. The device contained in the proof (namely a probabilistic integration by parts formula) will be used throughout the subsequent arguments.

Lemma III.1. Let $X$ and $Y$ be stochastically independent centered random vectors in $\mathbb{R}^{d}$. Suppose that $X$ (resp., $Y$ ) has score $\rho_{X}(X)$ (resp., $\rho_{Y}(Y)$ ) and Stein matrix $\tau_{X}(X)$ (resp., $\tau_{Y}(Y)$ ). For $0<t<1$, let $W_{t}=\sqrt{t} X+\sqrt{1-t} Y$ and $\Gamma_{t}$ be the covariance matrix of $W_{t}$. Then

$$
\begin{aligned}
& \rho_{W_{t}}\left(W_{t}\right)+\Gamma_{t}^{-1} W_{t} \\
= & E\left[\frac{t}{\sqrt{1-t}}\left(I d-\Gamma_{t}^{-1} \tau_{X}(X)\right) \rho_{Y}(Y)\right. \\
+ & \left.\frac{1-t}{\sqrt{t}}\left(I d-\Gamma_{t}^{-1} \tau_{Y}(Y)\right) \rho_{X}(X) \mid W_{t}\right]
\end{aligned}
$$

is a version of the score of $W_{t}$.

Proof. Let $\varphi \in C_{c}^{\infty}\left(\mathbb{R}^{d}\right)$ be a test function. Applying first (1) (with respect to $Y$ ) then (2) (with respect to $X$ ) we get

$$
\begin{aligned}
& \frac{1}{\sqrt{1-t}} E\left[\left(I d-\Gamma_{t}^{-1} \tau_{X}(X)\right) \rho_{Y}(Y) \varphi\left(W_{t}\right)\right] \\
& =-E\left[\left(I d-\Gamma_{t}^{-1} \tau_{X}(X)\right) \nabla \varphi\left(W_{t}\right)\right] \\
& =-\left(E\left[\nabla \varphi\left(W_{t}\right)\right]-\Gamma_{t}^{-1} \frac{1}{\sqrt{t}} E\left[X \varphi\left(W_{t}\right)\right]\right) .
\end{aligned}
$$


Likewise

$$
\begin{aligned}
& \frac{1}{\sqrt{t}} E\left[E\left[\left(I d-\Gamma_{t}^{-1} \tau_{Y}(Y)\right) \rho_{X}(X) \mid W_{t}\right] \varphi\left(W_{t}\right)\right] \\
& =-\left(E\left[\nabla \varphi\left(W_{t}\right)\right]-\Gamma_{t}^{-1} \frac{1}{\sqrt{1-t}} E\left[Y \varphi\left(W_{t}\right)\right]\right) .
\end{aligned}
$$

Hence

$$
\begin{aligned}
& E\left[E \left[\frac{t}{\sqrt{1-t}}\left(I d-\Gamma_{t}^{-1} \tau_{X}(X)\right) \rho_{Y}(Y)\right.\right. \\
& \left.\left.+\frac{1-t}{\sqrt{t}}\left(I d-\Gamma_{t}^{-1} \tau_{Y}(Y)\right) \rho_{X}(X) \mid W_{t}\right] \varphi\left(W_{t}\right)\right] \\
& =-E\left[\nabla \varphi\left(W_{t}\right)\right]+E\left[\Gamma_{t}^{-1} W_{t} \varphi\left(W_{t}\right)\right] \\
& =E\left[\left(\rho_{W_{t}}\left(W_{t}\right)+\Gamma_{t}^{-1} W_{t}\right) \varphi\left(W_{t}\right)\right],
\end{aligned}
$$

and the conclusion (4) follows.

It is immediate to extend (4) to an arbitrary number of summands.

Lemma III.2. Let $X_{i}, i=1, \ldots, n$ be independent random vectors with Stein matrices $\tau_{i}=\tau_{X_{i}}$ and score functions $\rho_{i}=\rho_{X_{i}}, i=1, \ldots, n$. For all $t=\left(t_{1}, \ldots, t_{n}\right) \in[0,1]^{d}$ such that $\sum_{i=1}^{n} t_{i}=1$ we define $W_{t}=\sum_{i=1}^{n} \sqrt{t_{i}} X_{i}$ and denote $\Gamma_{t}$ the corresponding covariance matrix. Then $\rho_{t}\left(W_{t}\right)+\Gamma_{t}^{-1} W_{t}=$ $\sum_{i=1}^{n} \frac{t_{i}}{\sqrt{t_{i+1}}} E\left[\left(I d-\Gamma_{t}^{-1} \tau_{i}\left(X_{i}\right)\right) \rho_{i+1}\left(X_{i+1}\right) \mid W_{t}\right]$ where we identify $X_{n+1}=X_{1}$ and $t_{n+1}=t_{1}$, and where we set $\rho_{t}=\rho_{W_{t}}$.

In [19] we use a version of (4) specialised to the case where $X$ has covariance $C$ and $Y=Z$ is a Gaussian random vector also with covariance $C$. Then $\Gamma_{t}=C$ and, setting $X_{t}=\sqrt{t} X+\sqrt{1-t} Z$, we get, for all $0<t<1$,

$$
\begin{aligned}
& \rho_{t}\left(X_{t}\right)+C^{-1} X_{t} \\
= & -\frac{t}{\sqrt{1-t}} E\left[\left(I d-C^{-1} \tau_{X}(X)\right) C^{-1} Z \mid X_{t}\right] .
\end{aligned}
$$

Taking squares and simplifying accordingly we obtain the following representations for the Fisher information and the standardised Fisher information of an arbitrary random vector with density.

Theorem III.3. Let $X$ be centered with covariance $I d$ independent of $Z$ standard Gaussian and $X_{t}=$ $\sqrt{t} X+\sqrt{1-t} Z$. For all $0<t<1, J\left(X_{t}\right)$ equals

$$
\begin{aligned}
& \frac{t^{2}}{1-t} E\left[E\left[\left(I d-C^{-1} \tau_{X}(X)\right) C^{-1} Z \mid X_{t}\right]\right. \\
& \left.\quad \times E\left[\left(I d-C^{-1} \tau_{X}(X)\right) C^{-1} Z \mid X_{t}\right]^{T}\right]+C^{-1},
\end{aligned}
$$

and $J_{s t}\left(X_{t}\right)$ equals

$$
\begin{aligned}
= & \frac{t^{2}}{1-t} \operatorname{tr}\left(C E \left[E\left[\left(I d-C^{-1} \tau_{X}(X)\right) C^{-1} Z \mid X_{t}\right]\right.\right. \\
& \left.\left.\times E\left[\left(I d-C^{-1} \tau_{X}(X)\right) C^{-1} Z \mid X_{t}\right]^{T}\right]\right) .
\end{aligned}
$$

Arguably, the main application of formula (7) provided in the present paper appears in Section $\mathbf{V}$ where we will deduce explicit bounds in the multidimensional entropic CLT. However, representation results such as (7) cover a much wider ground of applications, as they may lead in principle to new identities and new estimates in any domain where information functionals do appear. The reader is referred e.g. to [12] for a panoply of novel applications of formulae analogous to (7) to log-Sobolev and transport inequalities.

\section{Connection with a Formula of Guo, SHAMAI AND VERDÚ}

It was brought to our attention (by Oliver Johnson, personal communications) that representation (5) resembled, at least in principle, an identity for Fisher information discovered in [7]. The purpose of this section is to make the connection between the two approaches explicit.

Lemma IV.1 ([7, equation (56)]). Let $X$ be a centered random vector with covariance $C$ independent of $Z$ Gaussian with the same covariance as $X$. Then, for all $0<t<1$, the random vector $X_{t}=\sqrt{t} X+\sqrt{1-t} Z$ has a score $\rho_{t}\left(X_{t}\right)=-\frac{1}{1-t} C^{-1}\left(X_{t}-\sqrt{t} E\left[X \mid X_{t}\right]\right)$ and its Fisher information $J\left(X_{t}\right)$ equals $\frac{C^{-1}}{1-t}-$ $\frac{t C^{-1}}{(1-t)^{2}} E\left[\left(X-E\left[X \mid X_{t}\right]\right)\left(X-E\left[X \mid X_{t}\right]\right)^{T}\right] C^{-1}$.

Proof. Clearly, $X_{t}$ has a differentiable density with support $\mathbb{R}^{d}$. Let $\varphi \in C_{c}^{\infty}\left(\mathbb{R}^{d}\right)$ be a test function. Then

$$
\begin{aligned}
& E\left[C^{-1}\left(X_{t}-\sqrt{t} E\left[X \mid X_{t}\right]\right) \varphi\left(X_{t}\right)\right] \\
& =E\left[C^{-1}\left(X_{t}-\sqrt{t} X\right) \varphi\left(X_{t}\right)\right] \\
& =\sqrt{1-t} E\left[C^{-1} Z \varphi\left(X_{t}\right)\right]=(1-t) E\left[\nabla \varphi\left(X_{t}\right)\right] .
\end{aligned}
$$

Both claims then follow after straightforward computations.

Next, as in [7], we define $\operatorname{MMSE}(X, t)=$ $E\left[\left(X-E\left[X \mid X_{t}\right]\right)\left(X-E\left[X \mid X_{t}\right]\right)^{T}\right]$. Direct application of the above yields the following.

Proposition IV.2. If $A$ is a matrix we write $A^{2}$ for $A A^{T}$. Then $I d-\frac{1}{1-t} \operatorname{MMSE}(X, t) C^{-1}=$ $t C E\left[E\left[\left(I d-C^{-1} \tau_{X}(X)\right) C^{-1} Z \mid X_{t}\right]^{2}\right]$ so that $J_{s t}\left(X_{t}\right)=\frac{t}{1-t} \operatorname{tr}\left(I d-\frac{1}{(1-t)} \operatorname{MMSE}(X, t) C^{-1}\right)$.

Plugging this last identity into Lemma $\amalg$.4 shows that relative entropy is an integral of minimal squared error; this claim is equivalent (up to scaling) to [7, equation (57)]. 


\section{INFORMATION BOUNDS FOR SUMS OF RANDOM VECTORS}

In the sequel we suppose for simplicity that all random vectors are isotropic (i.e. have identity covariance matrix).

Theorem V.1. Let $X_{1}, \ldots, X_{n}$ be independent random vectors in $\mathbb{R}^{d}$ and suppose that the $X_{i}$ have Stein matrix $\tau_{i}\left(X_{i}\right)$ and score function $\rho_{i}\left(X_{i}\right)$. Let $W_{n}=\frac{1}{\sqrt{n}} \sum_{i=1}^{n} X_{i}$. Define $W_{n}^{(t)}=\sqrt{t} W_{n}+\sqrt{1-t} Z$, where $Z$ is an independent standard Gaussian random vector. Then $J_{s t}\left(W_{n}^{(t)}\right) \leq$ $\frac{t^{2}}{n^{2}(1-t)} \sum_{i=1}^{n} \operatorname{tr}\left(E\left[\left(I d-\tau_{i}\left(X_{i}\right)\right)\left(I d-\tau_{i}\left(X_{i}\right)\right)^{T}\right]\right)$ for all $0 \leq t \leq 1$.

Proof. First, by Jensen's inequality, we see that

$$
\begin{aligned}
& \operatorname{tr}\left(E \left[E\left[\left(I d-\tau_{W_{n}}\left(W_{n}\right)\right) Z \mid W_{n}^{(t)}\right]\right.\right. \\
& \left.\left.\quad \times E\left[\left(I d-\tau_{W_{n}}\left(W_{n}\right)\right) Z \mid W_{n}^{(t)}\right]^{T}\right]\right) \\
& \leq \operatorname{tr}\left(E\left[\left(I d-\tau_{W_{n}}\left(W_{n}\right)\right)\left(I d-\tau_{W_{n}}\left(W_{n}\right)\right)^{T}\right]\right) .
\end{aligned}
$$

Next, it is easy to prove (see [19] for a proof when $d=1)$ that $\tau_{W_{n}}\left(W_{n}\right)=\frac{1}{n} \sum_{i=1}^{n} E\left[\tau_{i}\left(X_{i}\right) \mid W_{n}\right]$ is a Stein matrix for $W_{n}$. Hence, by (7), $J_{s t}\left(W_{n}^{(t)}\right)$ is less than or equal to

$$
\begin{gathered}
\frac{t^{2}}{1-t} \operatorname{tr}\left(E\left[\left(I d-\tau_{W_{n}}\left(W_{n}\right)\right)\left(I d-\tau_{W_{n}}\left(W_{n}\right)\right)^{T}\right]\right) \\
\leq \frac{1}{n^{2}} \frac{t^{2}}{1-t} \operatorname{tr}\left(E \left[\left(\sum_{i=1}^{n}\left(I d-\tau_{i}\left(X_{i}\right)\right)\right)\right.\right. \\
\left.\left.\times\left(\sum_{i=1}^{n}\left(I d-\tau_{i}\left(X_{i}\right)\right)^{T}\right)\right]\right) .
\end{gathered}
$$

Independence of the $X_{i}$ as well as the fact that $E\left[I d-\tau_{i}\left(X_{i}\right)\right]=0$ allow to conclude.

In particular, if the $X_{i}$ are i.i.d. copies of $X$ then $J_{s t}\left(W_{n}^{(1 / 2)}\right) \leq$ $\frac{1}{2 n} \operatorname{tr}\left(E\left[\left(I d-\tau_{X}(X)\right)\left(I d-\tau_{X}(X)\right)^{T}\right]\right)$. By Cramer's theorem (see, e.g., [11]), convergence of $W_{n}$ to the Gaussian is equivalent to convergence of $W_{n}^{(1 / 2)}$, and Theorem V.1 provides rates of convergence (of order $1 / n$ ) of the Fisher information under the assumption that $X$ has a well-defined Stein matrix $\tau_{X}(X)$. A straightforward extension of [8, Lemma 1.21] to the multivariate setting shows that standardised information decreases along convolutions.

Lemma V.2. If $X$ and $Y$ are independent isotropic (i.e. identity covariance matrix) real-valued $d$ random vectors then $J_{s t}(\sqrt{t} X+\sqrt{1-t} Y) \leq$ $t J_{s t}(X)+(1-t) J_{s t}(Y)$.
Proof. Let $W_{t}=\sqrt{t} X+\sqrt{1-t} Y$. From definition (1) it is easy to see that $\rho_{t}(w)=$ $E\left[\sqrt{t} \rho_{X}(X)+\sqrt{1-t} \rho_{Y}(Y) \mid W_{t}=w\right]$. By definition $J\left(W_{t}\right)=\sum_{j=1}^{d} E\left[\left(\rho_{t}\left(W_{t}\right)\right)_{j}^{2}\right] \quad$ which, by Jensen's inequality, is smaller or equal to $t E\left[\sum_{j=1}^{d}\left(\rho_{X}(X)\right)_{j}^{2}\right]+(1-t) E\left[\sum_{j=1}^{d}\left(\rho_{Y}(Y)\right)_{j}^{2}\right]=$ $t J(X)+(1-t) J(Y)$, and the claim is proved for Fisher information. The extension to $J_{s t}$ is immediate.

In particular, from Lemma V.2, if $Z$ is standard Gaussian independent of $X$, then $J_{s t}\left(X_{t}\right) \leq$ $t J_{s t}(X)+(1-t) J_{s t}(Z)=t J_{s t}(X)$ for all $0 \leq$ $t \leq 1$ with $X_{t}=\sqrt{t} X+\sqrt{1-t} Z$, so that

$$
D(X \| Z) \leq \frac{1}{2} J_{s t}(X)
$$

by Lemma $\amalg .4$. Hence bounds on the standardised Fisher information translate directly into bounds on the relative entropy hereby providing, via Pinsker's inequality

$$
2 d_{T V}(X, Z) \leq \sqrt{2 D(X \| Z)},
$$

bounds on the total variation distance between the law of $X$ and the law of $Z$. From (8) we thus obtain rates of convergence in total variation which have the correct order (see e.g. [9], [2] for similar rates of convergence under the assumption of finite Poincaré constant).

Remark V.3. It is still largely an open question how the assumption of existence of a Stein matrix relates with more standard assumptions such as finiteness of the Poincaré constant. See e.g. [1], [19], [12] for discussions.

\section{STEIN REPRESENTATIONS FOR FISHER INFORMATION}

Our next lemma provides a new handle on conditional expectations which is also of independent interest.

Lemma VI.1 (Poly's lemma). Let $X$ and $Y$ be square-integrable random variables with mean $E[X]=0$. Then $E\left[(E[X \mid Y])^{2}\right]=$ $\sup _{\varphi \in \mathcal{H}(Y)}(E[X \varphi(Y)])^{2}$, where the supremum is taken over the collection $\mathcal{H}(Y)$ of functions $\varphi$ such that $E[\varphi(Y)]=0$ and $E\left[\varphi(Y)^{2}\right] \leq 1$.

Proof. First, by Cauchy-Schwarz,

$$
\begin{aligned}
& \sup _{\varphi \in \mathcal{H}(Y)}(E[X \varphi(Y)])^{2}=\sup _{\varphi \in \mathcal{H}(Y)}(E[E[X \mid Y] \varphi(Y)])^{2} \\
& \leq \sup _{\varphi \in \mathcal{H}(Y)} E\left[E[X \mid Y]^{2}\right] E\left[\varphi(Y)^{2}\right] \leq E\left[E[X \mid Y]^{2}\right]
\end{aligned}
$$


To prove the reverse inequality define $\varphi(y)=E[X \mid Y=y] / \sqrt{E\left[E[X \mid Y]^{2}\right]}$. Clearly $E[\varphi(Y)]=0$ and $E\left[\varphi(Y)^{2}\right] \leq 1$ so that $\varphi \in \mathcal{H}(Y)$ and $\sup _{\varphi \in \mathcal{H}(Y)}(E[X \varphi(Y)])^{2}$ is bigger than or equal to

$$
\begin{aligned}
& \left(E\left[X \frac{E[X \mid Y]}{\sqrt{E\left[E[X \mid Y]^{2}\right]}}\right]\right)^{2} \\
& =\frac{(E[X E[X \mid Y]])^{2}}{E\left[E[X \mid Y]^{2}\right]}=E\left[E[X \mid Y]^{2}\right] .
\end{aligned}
$$

Equality ensues.

We immediately deduce an original proof (not relying on Stein's method!) of a recently discovered fact (see e.g. [4], [14], [15]) that the Fisher information distance is dominated by expressions which appear naturally within the context of Stein's method.

Theorem VI.2 (Stein representation for relative Fisher information, $d=1$ ). Let $W_{n}=$ $\frac{1}{\sqrt{n}} \sum_{i=1}^{n} X_{i}$ where the $X_{i}$ are independent random variables with Stein factor $\tau_{i}\left(X_{i}\right)$ and score function $\rho_{i}\left(X_{i}\right)$. Then $\mathcal{J}\left(W_{n}\right)=$ $\sup _{\varphi \in \mathcal{H}\left(W_{n}\right)}\left(E\left[\varphi^{\prime}\left(W_{n}\right)-W_{n} \varphi\left(W_{n}\right)\right]\right)^{2}$.

Proof. We combine Lemma III.2 (in the special case $\left.t_{1}=t_{2}=\ldots=t_{n}=1 / n\right)$ and Lemma VI.1 to deduce that $n \mathcal{J}\left(W_{n}\right)$ is given by

$$
\begin{aligned}
& n E\left[\left(\rho_{n}\left(W_{n}\right)+W_{n}\right)^{2}\right] \\
= & E\left[\left(E\left[\sum_{i=1}^{n}\left(1-\tau_{i}\left(X_{i}\right)\right) \rho_{i+1}\left(X_{i+1}\right) \mid W_{n}\right]\right)^{2}\right] \\
= & \sup _{\varphi \in \mathcal{H}\left(W_{n}\right)}\left(\sum_{i=1}^{n} E\left[\left(1-\tau_{i}\left(X_{i}\right)\right) \rho_{i+1}\left(X_{i+1}\right) \varphi\left(W_{n}\right)\right]\right)^{2} \\
= & \sup _{\varphi \in \mathcal{H}\left(W_{n}\right)} E\left[\sqrt{n} \varphi^{\prime}\left(W_{n}\right)-\sum_{i=1}^{n} X_{i} \varphi\left(W_{n}\right)\right]^{2},
\end{aligned}
$$

and the conclusion follows.

$$
\begin{aligned}
& \text { Corollary VI.3 } \quad \text { (Stein representation } \\
& \text { for relative } \text { Fisher information). Let } \\
& W_{n}=\frac{1}{\sqrt{n}} \sum_{i=1}^{n} X_{i}=\left(\left(W_{n}\right)^{1}, \ldots,\left(W_{n}\right)^{d}\right)^{T} \\
& \text { where the } X_{i} \text { are independent } \quad d \text {-random } \\
& \text { vectors with } \quad \text { Stein } \quad \text { matrix } \tau_{i}\left(X_{i}\right) \text { and } \\
& \text { score function } \rho_{i}\left(X_{i}\right) \text {. Then } J_{s t}\left(W_{n}\right) \quad= \\
& \sum_{j=1}^{d} \sup _{\varphi \in \mathcal{H}\left(W_{n}\right)}\left(E\left[\partial_{j} \varphi\left(W_{n}\right)-\left(W_{n}\right)^{j} \varphi\left(W_{n}\right)\right]\right)^{2} .
\end{aligned}
$$

\section{ACKNOWLEDGMENT}

We are grateful to Oliver Johnson for providing the connexion with [7] and to Guillaume Poly for sharing his LemmaVI.1 with us. We thank the referees for their careful comments which helped improve the rendition of this work. Giovanni Peccati was partially supported by the Grant F1R-MTHPUL-12PAMP (PAMPAS) from Luxembourg University. Yvik Swan gratefully acknowledges support from the IAP Research Network P7/06 of the Belgian State (Belgian Science Policy).

\section{REFERENCES}

[1] H. Airault, P. Malliavin, and F. Viens. Stokes formula on the Wiener space and $n$-dimensional Nourdin-Peccati analysis. Journal of Functional Analysis, 258(5):1763-1783, 2010.

[2] K. Ball, F. Barthe, and A. Naor. Entropy jumps in the presence of a spectral gap. Duke Math. J., 119(1):41-63, 2003.

[3] K. Ball and V. Nguyen. Entropy jumps for random vectors with log-concave density and spectral gap. Preprint, arxiv:1206.5098v3, 2012.

[4] A.S. Barbour, O. Johnson, I. Kontoyiannis, and M. Madiman. Compound Poisson approximation via information functionals Electron. J. Probab, 15(42): 1344-1368, 2010.

[5] T. Cacoullos and V. Papathanasiou (1989): Characterizations of distributions by variance bounds. Statist. Probab. Letters 7, 351-356.

[6] L. H. Y. Chen, L. Goldstein, and Q.-M. Shao. Normal approximation by Stein's method. Probability and its Applications (New York). Springer, Heidelberg, 2011.

[7] D. Guo, S. Shamai, and S. Verdú. Mutual information and minimum mean-square error in gaussian channels. Information Theory, IEEE Transactions on, 51(4):12611282, 2005.

[8] O. Johnson. Information theory and the central limit theorem. Imperial College Press, London, 2004.

[9] O. Johnson and A. Barron. Fisher information inequalities and the central limit theorem. Probab. Theory Related Fields, 129(3):391-409, 2004.

[10] O. Johnson and Y. Suhov. Entropy and random vectors. J. Statist. Phys., 104(1-2):145-192, 2001.

[11] A. Kagan. A multivariate analog of the Cramer theorem on components of the Gaussian distributions. In Stability problems for stochastic models, pages 68-77. Springer, 1989.

[12] M. Ledoux, I. Nourdin and G. Peccati. Stein's method, logarithmic Sobolev and transport inequalities. arXiv preprint arXiv:1403.5855, 2014.

[13] E. L. Lehmann and G. Casella Theory of point estimation Springer Texts in Statistics. Springer-Verlag, New York, second edition, 1998.

[14] C. Ley and Y. Swan. Local Pinsker inequalities via Stein's discrete density approach. IEEE Trans. Info. Theory, 59(9):5584-4491, 2013.

[15] C. Ley and Y. Swan. Stein's density approach and information inequalities. Electron. Comm. Probab., 18(7):1-14, 2013.

[16] M. Madiman and A. Barron. Generalized entropy power inequalities and monotonicity properties of information. IEEE Transactions on Information Theory 53(7):23172329, 2007.

[17] I. Nourdin and G. Peccati. Normal approximations with Malliavin calculus : from Stein's method to universality. Cambridge Tracts in Mathematics. Cambridge University Press, 2012.

[18] I. Nourdin, G. Peccati and A. Réveillac. Multivariate normal approximation using Stein's method and Malliavin calculus. Ann. I.H.P. Proba. Stat., 46(1):45-58, 2010.

[19] I. Nourdin, G. Peccati, and Y. Swan. Entropy and the fourth moment phenomenon. Journal of Functional Analysis, 266, 3170-3207, 2013.

[20] I. Sason. On the entropy of sums of Bernoulli random variables via the Chen-Stein method. Information Theory Workshop (ITW) IEEE, 542-546, 2012. 